\title{
High Resolution Turbulence Treatment OF F/A-18 TAIL BUFFET
}

\author{
Scott A. Morton, ${ }^{*}$ Russell M. Cummings, ${ }^{\dagger}$ and Denis B. Kholodar ${ }^{\star}$ \\ United States Air Force Academy, Department of Aeronautics \\ 2410 Faculty Drive, Suite 108 \\ USAF Academy, CO 80840-6400, USA.
}

\begin{abstract}
Unsteady tail loads of the F/A-18 are computed using various turbulence models at an angle of attack consistent with buffet induced by leading-edge extension vortex breakdown. Comparison of these industry standard turbulence models with the Detached Eddy Simulation (DES) hybrid RANS-LES approach reveals the inadequacies of RANS methods and the ability of DES to reproduce the observed unsteadiness at these conditions. Computed vortex breakdown position and frequencies of the DES method are shown to be accurate by comparison to flight test and experimental results. Finally, comparison of the DES unsteady tail pressures with flight-test tail pressures reveal the ability of the method to accurately reproduce F/A-18 tail buffet loads and is ready for inclusion in a fluid-structure interaction method.
\end{abstract}

\section{INTRODUCTION}

$\mathrm{M}$ ANY of todays military vehicles exhibit vortex dominated flowfields. At a recent NATO Air Vehicle Technology conference, D. A. Lovell presented a review of "Military Vortices," where he discussed the declining research budget in this area and the importance of understanding the phenomena. He classified vortex flows into three categories, "those designed into a vehicle to improve performance, those which cannot be avoided and whose adverse affects must be minimized, and those that were not expected to occur." "He gives examples of many of these vortex dominated flowfields: tip vortices on wings having low sweep, leading edge extension vortices from the F-18 and F-16 aircraft, foreplanes on the Rafale, and flow over the MK-82 bomb, to name just a few. He also discusses the fact that governments are relying ever increasingly on the aerospace industry to perform research. Since the aerospace industry concentrates on cruise conditions for optimization of commercial aircraft, these vortical flowfields common in military aircraft are losing their place in research budgets. This is occurring at a time when the three largest US fighter development programs (F/A-18E/F, F/A-22, and F-35) incorporate twin tail configurations and high angle-of-attack maneuvering.

The F-18 High Angle of Attack Research Vehicle (HARV; see Fig. 1) has proven to be an excellent source of data for researchers working on high angle of attack flowfields. ${ }^{3,44}$ Extensive flight testing of the HARV has been conducted that provides a rich source of flow visualization, surface pressures, and aeroelastic information. The F/A-18 utilizes wing leading edge extensions (LEX) to generate vortices which enhance the wing lift, and the twin vertical tails are canted to intercept the strong vortex field and increase maneuverability. At large incidence, the LEX vortices break down upstream of the vertical tails, resulting in a loss of yaw control power and severe aeroelastic effects. ${ }^{5}$ The ultimate goal of computationally modeling the flowfield shown in Fig. 1 would be to accurately simulate the aeroelastic impact of the LEX vortices on the twin vertical tails. Previous predictions of the HARV flowfield include RANS computations with solid tails, ${ }^{43}$ Detached-Eddy Simulation predictions showing the impact of the breakdown region on the vertical tails ${ }^{44}$ and fully aeroelastic tails with laminar off-body flow and flow control methods for alleviating tail buffet. ${ }^{45}$ The current level of simulation technology, however, has not allowed for accurate prediction of vortex breakdown, and the unsteady flow downstream of breakdown, at flight Reynolds numbers. Because of this, researchers have spent time computing flows over simpler geometries, such as slender forebodies and delta wings, to improve their simulation capabilities. However, the advent of hybrid turbulence models may finally allow for the accurate prediction of full aircraft flowfields at high incidence.

\footnotetext{
*Associate Professor of Aeronautics, AIAA Associate Fellow.

$\dagger$ Distinguished Visiting Professor, AIAA Associate Fellow.

ॠ Research Engineer, AIAA Member.

This paper is declared a work of the US government and is not subject to copyright protection in the United States.
} 
While advances have taken place in areas such as grid generation and fast algorithms for solutions of systems of equations, CFD has remained limited as a reliable tool for prediction of inherently unsteady flows at flight Reynolds numbers. Current engineering approaches to prediction of unsteady flows are based on solution of the Reynolds-averaged Navier-Stokes (RANS) equations. The turbulence models employed in RANS methods necessarily model the entire spectrum of turbulent motions. While often adequate in steady flows with no regions of reversed flow, or possibly exhibiting shallow separation, it appears inevitable that RANS turbulence models are unable to accurately predict flows characterized by massive separation. Unsteady, massively separated flows are characterized by geometry-dependent and three dimensional turbulent eddies. These eddies, arguably, are what defeat RANS turbulence models from predicting flows of any complexity.

To overcome the deficiencies of RANS models for predicting massively separated flows, Spalart et al. ${ }^{18,19}$ proposed DetachedEddy Simulation (DES) with the objective of developing a numerically feasible and accurate approach combining the most favorable elements of RANS models and Large Eddy Simulation

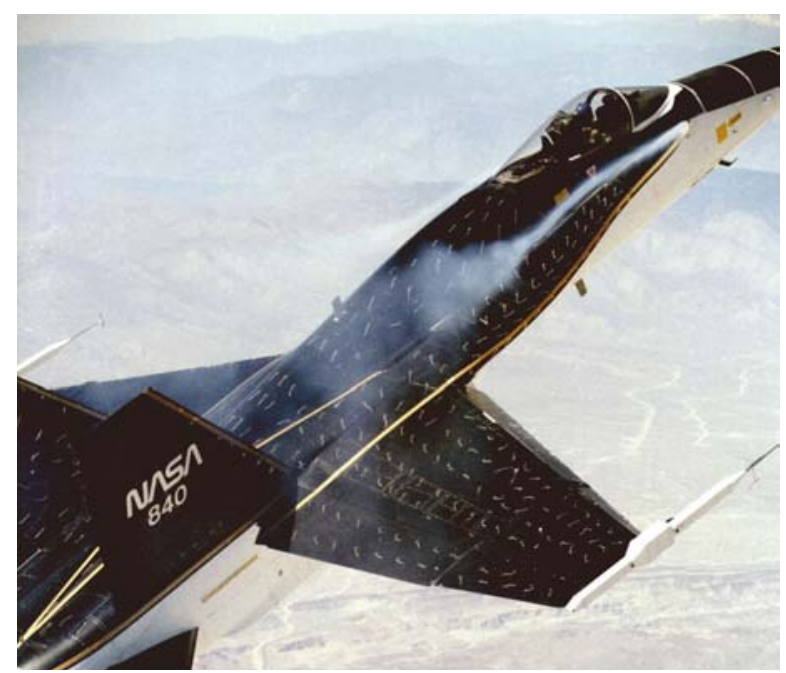

Figure 1. NASA F-18 High Angle of Attack Research Vehicle (HARV). Photo Courtesy of NASA Dryden. (LES). The primary advantage of DES is that it can be applied at high Reynolds numbers, as can Reynolds-averaged techniques, but DES also resolves geometry-dependent, unsteady threedimensional turbulent motions as in LES. The unstructured finite-volume solver Cobalt ${ }^{20}$ has been used in conjunction with DES successfully on a number of complex problems, including a supersonic base flow, ${ }^{21}$ delta wing vortex breakdown, ${ }^{22}$ a square with rounded corners, ${ }^{23}$ the F-15E at high angle of attack, ${ }^{24}$ and the F/A-18E with unsteady shock buffet. ${ }^{25}$

The specific aim of this work is to document the effects of applying detached eddy simulations to the F/A-18C at a condition consistent with vortex breakdown. Computations are made for the F/A-18C at $\alpha=30^{\circ}, M_{\infty}=0.2755$, and $\operatorname{Re}_{\bar{c}}=13 \times 10^{6}$ which determines the importance of turbulence model choice and the importance of using highly refined grids (including automatic mesh refinement) on the accurate prediction of complex vortical flowfields. Comparisons are made between two industry-standard unsteady Reynolds-averaged Navier-Stokes (U-RANS) methods, and the Spalart-Allmaras DES (SADES) method. The resulting predictions are compared with available flight test data for the F-18 HARV. Of this list, only SADES accurately predicts the post-breakdown flowfield, and therefore is capable of modeling tail buffet on the F/A-18C.

\section{NUMERICAL METHOD}

In this section a brief description of the numerical method is provided. Full details of the computational scheme and the solution method are presented in Ref. 20. The configuration of interest is the F/A-18C with leading edge flaps set to $-33^{\circ}$ and trailing edge flaps set to $0^{\circ}$, and the diverter slot with flow through the LEX is closed. This configuration closely matches the F-18 HARV for the chosen flight conditions.

Solutions were computed with the commercial version of Cobalt developed by Cobalt Solutions. ${ }^{20}$ Cobalt solves the unsteady, three-dimensional, compressible Navier-Stokes equations on a hybrid unstructured grid. The code has several choices of turbulence models, including Spalart Almaras (SA), and Menter's Shear Stress Transport (SST) RANS, as well as DES versions of SA and SST. All simulations were computed on unstructured meshes with prisms in the boundary layer and tetrahedra elsewhere on half-span surface geometries. The computational meshes were generated with the software packages GridTool $^{26}$ and VGRIDns. ${ }^{27}$

\section{A. Turbulence Models}

For simulation of turbulent flows, the governing equations are suitably averaged, yielding turbulent stresses that require a model. A Boussinesq approximation is invoked in the momentum equations and the turbulent eddy viscosity $\left(\mu_{t}\right)$ is used to relate the stresses to the strain rate. The turbulent heat flux is also modeled using a gradient-transport hypothesis, requiring specification of a turbulent thermal conductivity, $k_{t}$. The Reynolds analogy is applied and the turbulent heat flux is modeled using a constant turbulent Prandtl number of 0.9 . Using turbulent eddy viscosity and turbulent conductivity, the variable $\mu$ is replaced by $\left(\mu+\mu_{t}\right)$ and $k$ is replaced by $\left(k+k_{t}\right)$ in the governing equations. 


\section{B. MENTER's Shear STRESS Transport MODEL}

Menter's shear stress transport (SST) model is a hybrid $k-\varepsilon$ and $k-\omega$ turbulence model. Typical $k-\omega$ models are well behaved in the near wall region where low Reynolds number corrections are not required. However, they are generally sensitive to the freestream values of $\omega$. On the other hand, $k-\varepsilon$ models are relatively insensitive to freestream values, but behave poorly in the near wall region. Menter proposed a hybrid model. The SST model uses a parameter $F_{1}$ to switch from $k-\omega$ to $k-\varepsilon$ in the wake region to prevent the model from being sensitive to freestream conditions. The governing differential equations, including a compressibility correction, are given by:

$$
\begin{aligned}
& \frac{D(\rho k)}{D t}=\tau_{i j} \frac{\partial u_{i}}{\partial x_{j}}+\left(1-F_{1}\right) \overline{p^{\prime \prime} d^{\prime \prime}} \\
& -\frac{\rho k^{3 / 2}}{l_{k-\omega}}+\frac{\partial}{\partial x_{j}}\left[\left(\mu+\sigma_{k} \mu_{t}\right) \frac{\partial k}{\partial x_{j}}\right]
\end{aligned}
$$

where

and

$$
l_{k-\omega}=\frac{k^{1 / 2}}{\beta^{*}\left[1+\alpha_{1} M_{t}^{2}\left(1-F_{1}\right)\right] \omega}
$$

$$
\begin{aligned}
& \frac{D(\rho \omega)}{D t}=\frac{\gamma \rho}{\mu_{t}} \tau_{i j} \frac{\partial u_{i}}{\partial x_{j}}+\left(1-F_{1}\right) \beta^{*} \alpha_{1} M_{t}^{2} \rho \omega^{2} \\
& -\beta \rho \omega^{2}+\frac{\partial}{\partial x_{j}}\left[\left(\mu+\sigma_{\omega} \mu_{t}\right) \frac{\partial \omega}{\partial x_{j}}\right] \\
& +2 \rho\left(1-F_{1}\right) \sigma_{\omega 2} \frac{1}{\omega} \frac{\partial k}{\partial x_{j}} \frac{\partial \omega}{\partial x_{j}}-\left(1-F_{1}\right) \frac{\overline{p^{\prime \prime} d^{\prime \prime}}}{v_{t}}
\end{aligned}
$$

where the pressure dilatation term is

$$
\overline{p^{\prime \prime} d^{\prime \prime}}=-\alpha_{2} \tau_{i j} \frac{\partial u_{i}}{\partial x_{j}} M_{t}^{2}+\alpha_{3} \rho \varepsilon M_{t}^{2},
$$

and $M_{t}^{2}=\frac{2 k}{a^{2}}$ is termed the turbulent Mach number. The closure coefficients for the compressible corrections are

$$
\alpha_{1}=1.0 \quad \alpha_{2}=0.4 \quad \alpha_{3}=0.2 .
$$

Compressible corrections were in the SST formulation. The switching function, $F_{1}$, can be computed using

where

$$
F_{1}=\tanh \left(\arg _{1}^{4}\right)
$$

$$
\arg _{1}=\min \left(\max \left(\frac{\sqrt{k}}{0.9 \omega y}, \frac{500 \mu}{\rho \omega y^{2}}\right), \frac{4 \rho \sigma_{\omega 2} k}{C D_{k \omega} y^{2}}\right),
$$

and

$$
C D_{k \omega}=\max \left[2 \rho \sigma_{\omega 2} \frac{1}{\omega} \frac{\partial k}{\partial x_{i}} \frac{\partial \omega}{\partial x_{i}}, 10^{-20}\right] .
$$

The switching function also determines the value of the model constants. If $\phi_{1}$ represents a generic constant of the $k-\omega$ equations, and $\phi_{2}$ represents the same constant for the $k-\varepsilon$ equations, then the model constants used in the combined method are determined by

$$
\phi=F_{1} \phi_{1}+\left(1-F_{1}\right) \phi_{2} .
$$

The shear stress transport modifications enhance the model's accuracy for separated flows by limiting the turbulent shear stress to $\rho a_{1} k$ where $a_{1}=0.31$. The turbulent viscosity is given by 


$$
\mu_{t}=\frac{\rho a_{1} k}{\max \left(a_{1} \omega, \Omega F_{2}\right)},
$$

where $\Omega$ is the absolute value of vorticity. The function $F_{2}$ is included to prevent singular behavior in the freestream where $\Omega$ goes to zero. $F_{2}$ is given by

$$
\begin{gathered}
F_{2}=\tanh \left(\arg _{2}^{2}\right), \\
\arg _{2}=\max \left(\frac{2 \sqrt{k}}{0.09 \omega y}, \frac{400 v}{\omega y^{2}}\right) .
\end{gathered}
$$

The model constants were recalibrated for the shear stress transport modifications and the only change was to $\sigma_{k 1}$. The following are the coefficients for the hybrid model

$$
\begin{gathered}
\text { Set 1: }(k-\omega) \\
\sigma_{k 1}=0.85 \quad \sigma_{\omega 1}=0.5 \quad \beta_{1}=0.0750 \\
\beta^{*}=0.09 \quad \kappa=0.41 \quad \gamma_{1}=\frac{\beta_{1}}{\beta^{*}}-\frac{\sigma_{\omega 1} \kappa^{2}}{\sqrt{\beta^{*}}} \\
\sigma_{k 2}=1.0 \quad \sigma_{\omega 2}=0.856 \quad \beta_{2}=0.0828 \\
\beta^{*}=0.09 \quad \kappa=0.41 \quad \gamma_{2}=\frac{\beta_{2}}{\beta^{*}}-\frac{\sigma_{\omega 2} \kappa^{2}}{\sqrt{\beta^{*}}}
\end{gathered}
$$

\section{Spalart-Allmaras Turbulence Model}

The Spalart-Allmaras ${ }^{18}$ (SA) one equation model solves a single partial differential equation for a working variable $\tilde{v}$ which is related to the turbulent viscosity. The differential equation is derived by "using empiricism and arguments of dimensional analysis, Galilean invariance and selected dependence on the molecular viscosity." "The model includes a wall destruction term that reduces the turbulent viscosity in the laminar sublayer. The model takes the form,

$$
\begin{aligned}
\frac{D \widetilde{v}}{D t} & =c_{b 1} \widetilde{S} \widetilde{v}-c_{w 1} f_{w}\left[\frac{\widetilde{v}}{d}\right]^{2} \\
& +\frac{1}{\sigma}\left[\nabla \cdot((v+\widetilde{v}) \nabla \widetilde{v})+c_{b 2}(\nabla \widetilde{v})^{2}\right]
\end{aligned}
$$

The turbulent kinematic viscosity is obtained from,

$$
v_{t}=\frac{\mu_{t}}{\rho}=\widetilde{v} f_{v 1}, \quad f_{v 1}=\frac{\chi^{3}}{\chi^{3}+c_{v 1}^{3}}, \quad \chi \equiv \frac{\widetilde{v}}{v}
$$

where $S$ is the magnitude of the vorticity given by

$$
S=|\omega|=|\nabla \times(u \hat{i}+v \hat{j}+w \hat{k})|
$$

and the modified vorticity is,

$$
\tilde{S} \equiv S+\frac{\tilde{v}}{\kappa^{2} d^{2}} f_{v_{2}}, \quad f_{v_{2}}=1-\frac{\chi}{1+\chi f_{v 1}},
$$

where $d$ is the distance to the closest wall. The wall destruction function $f_{w}$ is,

and

$$
f_{w}=g\left[\frac{1+c_{w 3}^{6}}{g^{6}+c_{w 3}^{6}}\right]^{\frac{1}{6}}
$$

$$
g=r+c_{w 2}\left(r^{6}-r\right), \quad r \equiv \frac{\widetilde{v}}{\widetilde{S} \kappa^{2} d^{2}} .
$$


The turbulent viscosity is obtained from the turbulent kinematic viscosity by $\mu_{t}=\rho v_{t}$. The model coefficients are,

$$
\begin{array}{ccc}
c_{b 1}=0.1355 & \sigma=2 / 3 & c_{b 2}=0.622 \\
\kappa=0.41 & c_{w 1}=c_{b 1} / \kappa^{2}+\left(1+c_{b 2}\right) / \sigma & c_{w 2}=0.3 \\
c_{w 3}=2 & c_{v 1}=7.1 &
\end{array}
$$

\section{DETACHED-EDDY SimULATION}

Detached-Eddy Simulation (DES) was proposed by Spalart et al. ${ }^{28}$ The motivation for this approach was to combine largeeddy simulation (LES) with the best features of Reynolds-averaged Navier-Stokes (RANS) methods. RANS methods have demonstrated an ability to predict attached flows very well with a relatively low computational cost. LES methods have demonstrated an ability to compute seperated flowfields accurately, but at a tremendous cost for configurations with boundary layers. Spalart's DES method is a hybrid of LES and RANS, which combines the strengths of both methods.

The DES model was originally based on the Spalart-Allmaras one equation RANS turbulence model (detailed above). The wall destruction term presented above is proportional to $(\tilde{v} / d)^{2}$, where $\mathrm{d}$ is the distance to the wall. When this term is balanced with the production term, the eddy viscosity becomes proportional to $\hat{S} d^{2}$ where $\hat{S}$ is the local strain rate. The Smagorinski LES model varies its sub-grid scale (SGS) turbulent viscosity with the local strain rate, and the grid spacing: $v_{S G S} \propto \hat{S} \Delta^{2}$, where $\Delta=\max (\Delta x, \Delta y, \Delta z)$. If $d$ is replaced with $\Delta$ in the wall destruction term, the S-A model will act as a Smagorinski LES model.

To exhibit both RANS and LES behavior, $d$ in the SA model is replaced by

$$
\tilde{d}=\min \left(d, C_{D E S} \Delta\right) \text {. }
$$

When $d<<\Delta$, the model acts in a RANS mode and when $d>>\Delta$ the model acts in a Smagorinski LES mode. Therefore the model switches into LES mode when the grid is locally refined.

DES was implemented in an unstructured grid method by Forsythe et al. ${ }^{29}$ They determined the $C_{D E S}$ constant should be 0.65 , consistent with the structured grid implementation of Spalart et al. ${ }^{28}$ when the grid spacing $\Delta$ was taken to be the longest distance between the cell center and all of the neighboring cell centers.

A Newton sub-iteration method is used in the solution of the system of equations to improve time accuracy of the pointimplicit method and approximate Jacobians. In the calculations presented below, a typical number of three Newton subiterations is used for all time-accurate cases.

\section{E. GRID GENERATION}

Spalart ${ }^{19}$ described the process of grid design and assessment for DES, defining important regions of the solution and offering guidelines for grid densities within each region. The "Young-Person's Guide"19 (YPG) forms a basis for interpretation of many of the results presented below. One of the traditional motivations for using unstructured grids has been the ability to rapidly create grids around complex geometries. There are other positive attributes of unstructured grids that are relevant to DES. Most notably, it is possible to concentrate points in the region of interest (i.e. the vortex core or aft of breakdown) and rapidly coarsen the grid away from these areas. This region of interest was termed the "focus region" in the YPG. Another advantage exploited in the present study is the isotropic cells generated in the LES region by most unstructured grid generation packages. The YPG reference describes the desirability of having isotropic grid cells in the focus region in which unsteady, time-dependent, features are resolved. For this reason, unstructured grids are good candidates for use in DES because near isotropy of the grid cells in the LES region is assured by most grid generation packages.

Morton et al. ${ }^{30}$ applied the YPG guidelines to three massively separated flows of interest: forebody in a cross-flow, flow over a delta wing at $27^{\circ}$ angle of attack, and the flow over an F-15E at $65^{\circ}$ angle of attack. In the latter two cases an extensive grid sensitivity study was performed by systematically varying the grid by a scale parameter allowing a very consistent analysis of grid effects when using the DES method of computing massively sesparated flows. A further refinement of the delta wing grids was presented in Ref. 31 as well as the first use of adaptive mesh refinement with DES.

Another important grid technology that is particularly well suited for DES is adaptive mesh refinement. Pirzadeh ${ }^{32}$ presented a method based on a tetrahedral unstructured grid technology developed at NASA Langley Research Center with application to two configurations with vortex dominated flowfields. The large improvement of the adapted solutions in capturing vortex flow structures over the conventional unadapted results was demonstrated by comparisons with wind tunnel data. Pirzadeh showed the numerical prediction of these vortical flows was highly sensitive to the local grid resolution and he also stated that grid adaptation is essential to the application of CFD to these complicated flowfields. His most successful computations were performed using an inviscid method due to the inadequacies of standard turbulence models in computing these complicated flowfields. Pirzadeh's method is applied to the F/A-18C in the current study. A mean flow solution on a baseline grid is used to create an adaptively refined mesh and the new grid used with DES to compute the unsteady flowfield for these two configurations. All meshes of the current study produced average $\mathrm{y}^{+}$values less than 1. 


\section{F. SUMMARY OF THE PROPOSED METHOD} follows:

The proposed method for simulating aircraft at flight Reynolds numbers in conditions of massively separated flow is as

1. Use a time-accurate unstructured-grid solver to allow rapid turn around of grids on complex configurations -- the solution must have at least second-order spatial and temporal accuracy.

2. Use DES as the underlying turbulence treatment to obtain accurate unsteady loads and mean quantities - this requires a low dissipation solver.

3. Use Adaptive Mesh Refinement (AMR) to improve grid resolution in critical areas with nonlinear flowfield phenomena. Following this method has been shown to accurately predict vortex flowfields with vortex breakdown. ${ }^{44}$



a)

Figure 7. a) Top View of the Baseline Grid (3.9 million cells). b) Baseline grid of 3.6 million cells (left) and AMR grid of 3.9 million cells (right) at a station 450 inches aft of the origin. Darkened regions above the LEX show adapted mesh refinement of the vortex.

\section{RESULTS}

This section presents results of the numerical simulations for the F/A-18C with comparison to the NASA HARV flight-test data. The results section will be separated into subsections for comparison of solutions with various turbulence models and then comparison of SADES solutions with flight-test data.

All F/A-18C cases were run at $30^{\circ}$ angle-of-attack, a Mach number of 0.2755 , and a standard day altitude of 20,000 feet. The resulting Reynold's number was 13 million based on the mean aerodynamic chord of the aircraft (12 ft). The baseline grid of 3.6 million cells was generated with VGRIDns after starting from a geometry file provided by Cobalt Solutions LLC from their F/A-18C work. Unsteady SADES turbulence model simulations were performed using the baseline grid. A time-averaged SADES solution was used to produce an AMR grid with 3.9 million cells by following the approach outlined in Ref. 44. All time-accurate simulations were run for over 10,000 iterations with second-order temporal and spatial accuracy, three Newton sub-iterations, and a time step of 0.0005 seconds. The chosen time step results in a time step non-dimensionalized by the freestream velocity and mean aerodynamic chord of 0.0012 . This characteristic time step was found adequate in previous studies of vortex breakdown and massively separated flows. $2,24,44$

Figure 7a depicts a top view of the surface mesh and $7 \mathrm{~b}$ depicts a cross-plane at a station 450 inches aft of the origin for both the baseline grid and the AMR grid. The AMR grid has enhanced resolution in the core of the LEX vortex and over the wing. These enhanced grid regions are due to the AMR based on a vorticity iso-surface corresponding to separation regions at these locations and due to the vorticity in the LEX vortex core. 


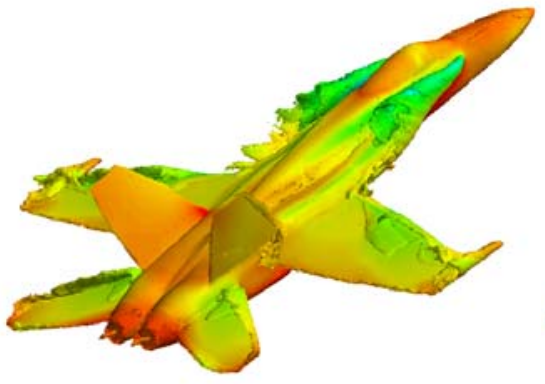

a) SST

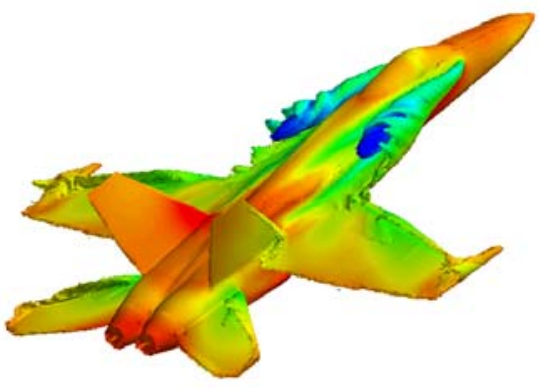

b) $\mathbf{S A}$

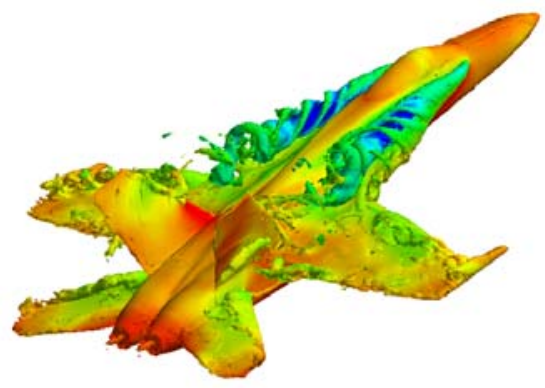

c) SADES

Figure 8. Isometric views of the $\mathrm{F} / \mathrm{A}-18 \mathrm{C}$ at $\alpha=30^{\circ}, \operatorname{Re}_{\mathrm{c}}=13 \times 10^{6}$, leading edge flaps set to $-33^{\circ}$, trailing edge flaps set to $0^{\circ}$, with no diverter slot present: a) SST turbulence model, b) SA turbulence model, and c) SADES turbulence model.

\section{A. Comparison of Various Turbulence Models}

Solutions were computed using the SST, SA, and SADES turbulence models to determine their effect on the flowfield. Solutions for all three methods were computed using the same grid, time step, and number of sub-iterations to provide a consistent comparison. Figure 8 a-c depicts snapshots of solutions for each method with the surface colored by pressure and an iso-surface of vorticity shown. The chosen vorticity level for the isosurface and the pressure colormap are held fixed. Although the snapshots are not necessarily synchronized in time, the overall differences are striking. The SADES solution (Fig. 8c) produces a much more detailed view of the simulation since it is able to capture much finer flowfield scales. The SST (Fig. 8a) and SA (Fig. 8b) models are unable to capture the proper post-breakdown behavior or the leading-edge separation regions of the wing, horizontal, and vertical tails. It is also apparent that the SST LEX vortex pressure footprint on the surface is significantly different than either the SA or SADES solutions. The low pressure region represented by a dark green color is greatly reduced in size on the SST solution. The SADES solution is also capturing the vortical sub-structures around the

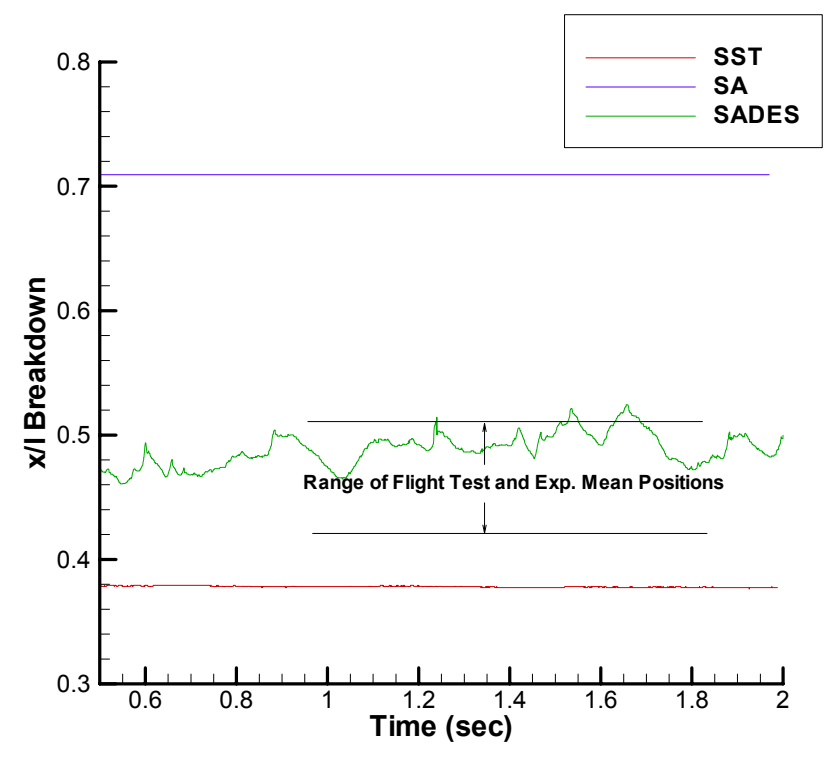

Figure 9. Time histories of the streamwise coordinate of vortex breakdown referenced to the vehicles nose and scaled by the length for the SST, SA, and SADES methods.

\begin{tabular}{|l|c|c|c|c|}
\hline & $\mathbf{R e} \overline{\mathbf{c}}$ & $\begin{array}{c}\text { Model scale, } \\
\text { percent }\end{array}$ & Fluid medium & Ret. \\
\cline { 2 - 5 } - Flight & $\mathbf{8}$ to $\mathbf{1 3 \times 1 0 ^ { 6 }}$ & & Air & $\mathbf{1 8}$ \\
ב DTRC & $\mathbf{1 . 7 5 \times 1 0 ^ { 6 }}$ & 6 & Air & $\mathbf{4 , 1 8}$ \\
O BART & $\mathbf{1 . 6 0 \times 1 0 6}$ & 3 & Air & $\mathbf{1 8}$ \\
A LSWT & $\mathbf{3 . 6 0 \times 1 0 ^ { 5 }}$ & 12 & Air & 18 \\
D FVF & $1.26 \times 10^{4}$ & 3 & Water & 18 \\
\hline
\end{tabular}

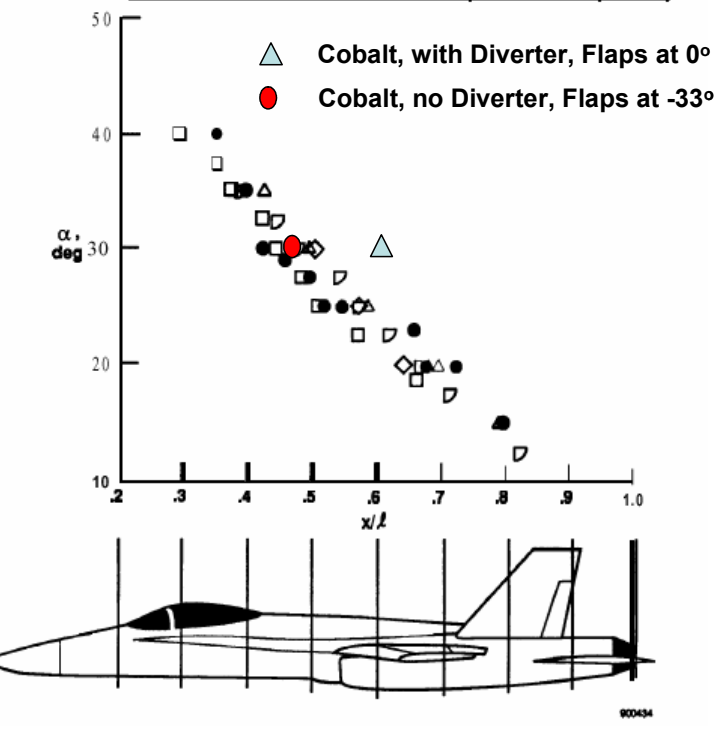

Figure 10. Streamwise LEX vortex breakdown position as a function of angle of attack, extracted from Ref. 47 SADES mean vortex breakdown position in red. 


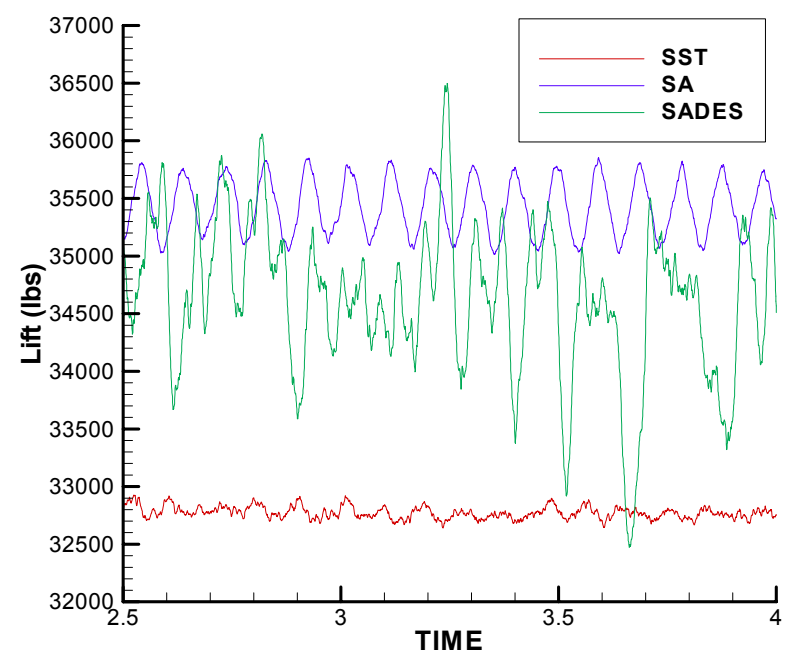

a)

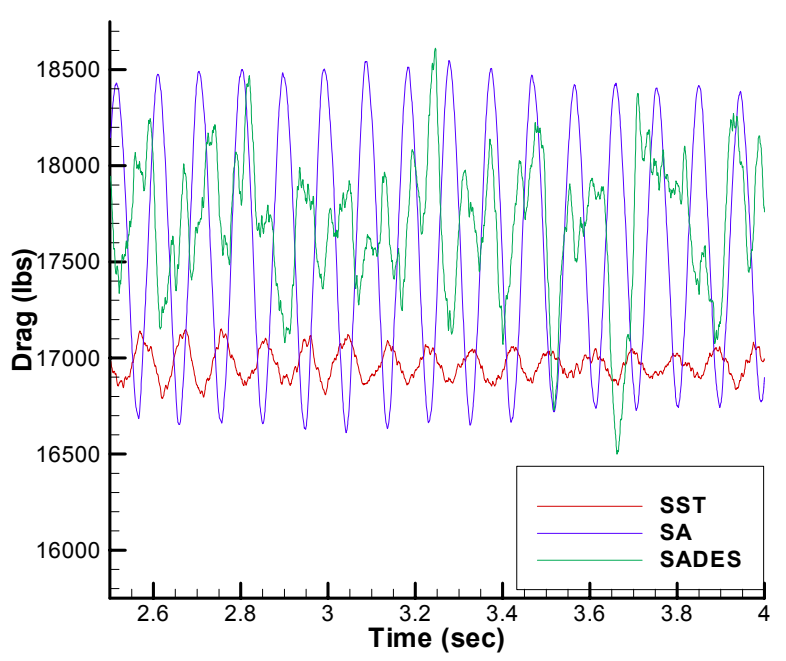

b)

Figure 11. Time histories of the a) Lift force and b) Drag force in lbs for the SST, SA, and SADES methods

A common definition of vortex breakdown is the location where the streamwise velocity component is zero in the core. The coordinates of this point along the core were tracked in time for each of the methods, SST, SA, and SADES. Figure 9 depicts the time histories of the three methods as well as the flight test and experiment maximum and minimum mean values of vortex breakdown presented in Ref. 47. Three things are obvious from Fig. 9. First, the amplitude of oscillation for the SST and SA models is almost negligible compared to the SADES simulation. Second, the SST solution predicts breakdown far upstream of the flight test or experimental values whereas the SA solution predicts the breakdown location downstream of the flight test and experimental results. Third, the SADES solution gives a mean value of vortex breakdown location well within the flight test and experimental data. It should also be noted that the computed nondimensional primary frequency of the breakdown oscillation is 0.2 in the range of frequencies commonly found in the literature ${ }^{46}$ for vortex breakdown. This inability of commonly used turbulence models to accurately compute a solution with breakdown is well documented in the literature and is due to the large amount of eddy-viscosity these models put into the core of vortices. ${ }^{22}$ Several researchers have proposed fixes to these turbulence models by incorporating some form of a rotation correction. The disadvantage of this approach is the fact the simulation will still be operating in a RANS mode and compute solutions that are relatively steady post-breakdown as opposed to an LES approach that resolves the eddies that produce the unsteadiness. It is clear in Fig's 8 and 9 that the SADES method does not suffer from the same problem as the RANS methods due to the fact that eddy viscosity is computed based on sub-grid scale turbulence, automatically minimizing the amount of spurious eddy-viscosity that is placed in the core of vortices.

Figure 10 is a well known plot in the literature of the streamwise location of the LEX vortex breakdown as a function of angle-of-attack ${ }^{47}$. The current solutions fall in the range of flight tests and experiments plotted at $30^{\circ}$ angle of attack. The previous comparisons of the method with the flight test and experimental data was poor due to the incorrect flap settings and diverter slot being uncovered. ${ }^{44}$

The effect of the various turbulence methods on computing the loads for these massively separated flows is demonstrated in Figs. 11a and b. Figure 11a shows the lift force on the vehicle as a function of time for the SST, SA, and SADES methods. As in the case of vortex breakdown position, the lift force computed with SADES has a significant amount of frequency content when compared to SST or SA. Also, the effect of the SST method predicting vortex breakdown far upstream is seen in a loss of lift. Fig. 11b shows similar trends in the drag force. The interesting feature to note in Fig. $11 \mathrm{~b}$ is the large

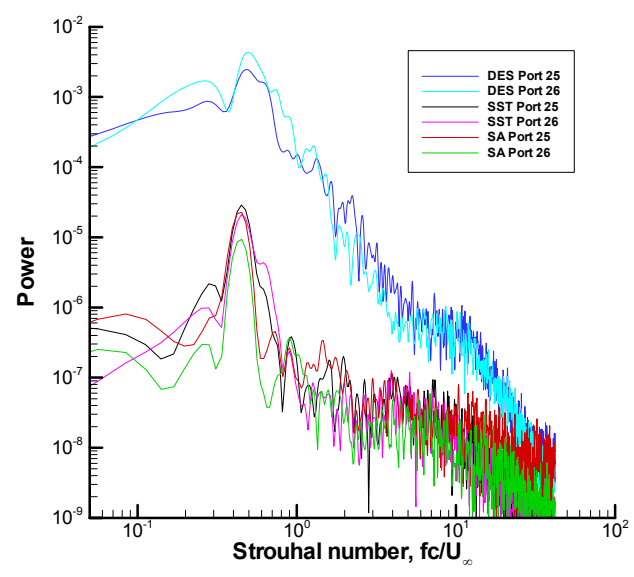

Figure 12. Power Spectrum Density Comparison for SST, SA, and SADES Turbulence Models. 
amplitude of oscillation in drag force computed with the SA model.

To determine the effect of using different turbulence models on the computed solutions frequency content, data was obtained at a "virtual" pressure port on the inboard and outboard sides of the vertical tail at $10 \%$ back from the leading edge and at $85 \%$ of the span. A power spectrum density (PSD) analysis was performed using MATLAB on the data from these pressure ports and presented in Fig. 11, with the inboard port numbered 25 and the outboard port numbered 26 (matching the flight test numbering system). The frequency output of the PSD was scaled by the mean aerodynamic chord and freestream velocity to produce the non-dimensional Strouhal frequency. The power was scaled by the number of points in the time history. Since the number of points and the time step was consistent between the three methods, the magnitude of the power from one method relative another method becomes significant. All three of the methods show a peak Strouhal frequency of approximately 0.5. The SADES method shows a three-orders of magnitude increase in the power for the majority of the frequencies up to a Strouhal number of 3 . Also the typically seen $-5 / 3$ slope roll off in power is at a much lower frequency in the SST and SA simulations than the SADES simulations. This shows the ability of the SADES method to resolve a greater frequency spectrum.

\section{B. COMPARISON TO NASA F-18 HARV FLIGHT TEST DATA}

This section presents comparison of the computed SADES solutions with F-18 HARV flight test data from NASA Dryden. The HARV was instrumented with 32 kulite pressure sensors, half on the inboard and half on the outboard sections of the right vertical tail (Fig. 13). The kulite pressures were stored every $30 \mathrm{~ms}$ as a function of time. The available pressures were stored relative to a reference pressure that is unfortunately unknown. The lack of known reference pressures allowed only frequency comparisons rather than frequency and amplitude comparisons of the SADES data with flight test data. Pressure ports of Fig. 13 circled in red are those used for comparison with the SADES simulations.

The flight test and SADES simulation port pressures were analyzed with MATLAB's PSD function as discussed in an earlier section. Since the flight test data has a different time step and period of time $(40 \mathrm{sec})$, the power resulting from a PSD analysis will not be a one to one match but the frequencies and characteristic shapes of the PSD should match. All 32 pressure ports were analyzed but only a representative set are shown. The ports chosen for presentation provide a series of data along the leading edge and a variation in span, as well as a series of chord locations at a particular span. Figures 14 a-d depict the comparison of SADES and flight test data. Figure 14a shows the PSD data for flight test and SADES simulation for ports 1 and 2, 14b shows ports 13 and 14, 14c shows ports 17 and 18 , and $14 \mathrm{~d}$ shows ports 25 and 26. In all cases, the frequency content shows quite good comparison between the flight test and SADES simulations. All of the ports show a wide peak amplitude range corresponding to Strouhal numbers between 0.45 and 0.8 for both flight test and SADES simulations. This frequency range corresponds to pressure sweeps over the tail surface observed in a movie clip of the SADES simulation. Unfortunately, the published first bending mode is at a Strouhal number of approximately 0.66 explaining why the tail is so aeroelastically active at this flight condition. Most of the ports also show matches in slopes of the PSD for the Strouhal range of $1-10$. Ports 1 and 2 compare least favorably in the higher Strouhal range $(1-10)$. These ports are located in a region of very complicated flow structures (as seen in Fig. 8c). An increase in grid resolution in this area is probably warranted. It is also interesting to note that when the flight test curves for each port lie on top of each other this is true for the SADES solutions as well (Figs. 14c and d), and when the flight test curves are separated they are separated by approximately the same amount in the SADES solutions (Fig. 14b). A consistency is noted in the level of power between inboard and outboard ports for both flight test and SADES, i.e. when the inboard port has a higher power for flight test that is true as well for the SADES simulation. Finally, when the curves cross, this occurs at approximately the same frequency for flight test and SADES (Fig. 14d). The overall comparison of frequency content is remarkably good for the SADES solutions, demonstrating the utility of the method for tail buffet computations at flight Reynolds numbers. 


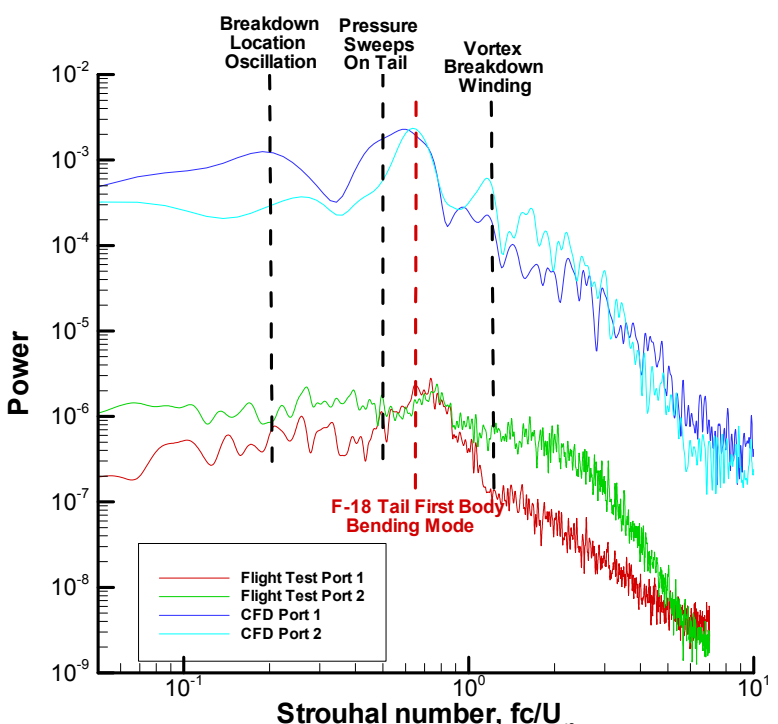

a)

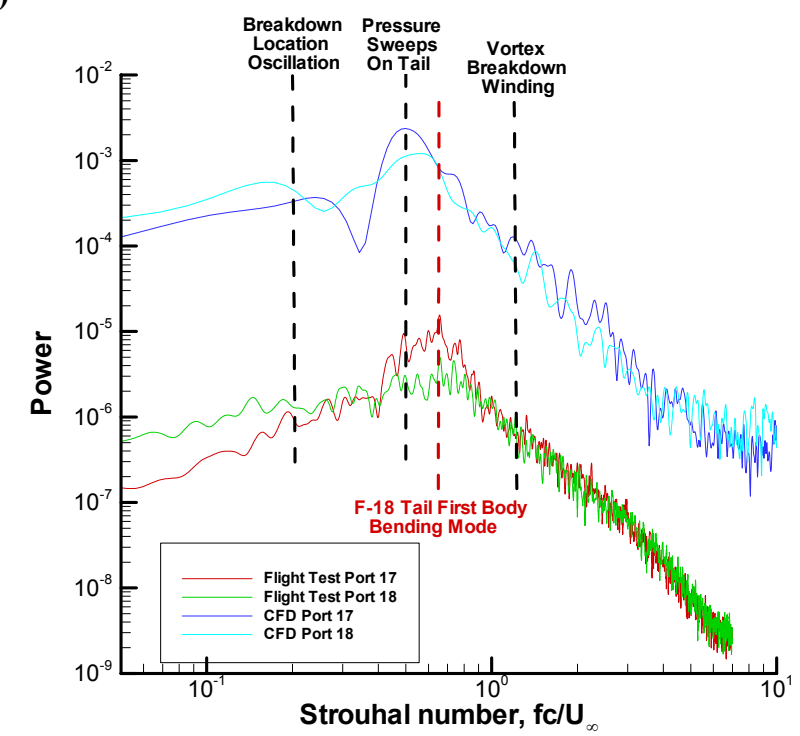

c)

Figure 14. Comparison of Power Spectrum Density from Flight Test and DES Prediction for a) Ports 1 \& 2, b) Ports 13 \& 14, c) Ports 17 \& 18, and d) Ports 25 \& 26.

\section{CONCLUSION \& RECOMMENDATIONS}

The combined SADES and AMR grid approach was used to compute solutions of the F/A-18C in a configuration closely matching the F-18 HARV flight test vehicle. The SST and SA turbulence methods were shown to be inconcsistent with each other and compute vortex breakdown locations either far upstream or downstream of the SADES method, as well as, flight test and experimentally obtained vortex breakdown positions. This mismatch in vortex breakdown mean position results in vastly different loads characteristics. In addition, the frequency content of tail pressures were more than three orders of magnitude lower in power than the corresponding SADES solutions causing concern for their use in fluid-structure interaction simulations. The SADES solutions were then compared to flight test kulite pressures from the F-18 HARV vertical tails. The frequencies obtained with the SADES method matched the kulite pressures remarkably well for both particular frequencies, and the overall character of the PSDs. This level of comparison shows the usefulness of this fluid-dynamics method for inclusion in a fluid-structure interaction method for these massively separated flow regimes. 


\section{ACKNOWLEDGMENTS}

The authors would like to say thanks to Dave Fisher and Al Bowers of the NASA Dryden Flight Research Center for their invaluable assistance with the F-18 HARV flight test data, as well as Maj James Forsythe for modifying the F/A-18C grid during his USAF Reserve duty. This work could not have been accomplished without the computational support of the Maui High Performance Computing Center of HPCMO and the USAFA Modeling \& Simulation Resource Center. Finally, the authors would like to express their gratitude for the support of this project by Dr John Schmisseur of AFOSR.

\section{REFERENCES}

${ }^{1}$ Lovell, D A, "Military Vortices,” NATO Research \& Technology Organization Applied Vehicle Technology Panel Meeting, Norway,
7-11 May 2001.
${ }^{2}$ Morton, S.A., Forsythe, J.R., Mitchell, A.M., and Hajek, D., "DES and RANS Simulations of Delta Wing Vortical Flows," AIAA Paper 2002-0587, Jan. 2002.

${ }^{3}$ Lee, B.H.K., "Vertical Tail Buffeting of Fighter Aircraft,” Progress in Aerospace Sciences, Vol. 36, No. 3-4, 2000 , pp. $193-279$.

${ }^{4}$ Fisher, D., Del Frate, J.H., and Zuniga, F.A., "Summary of In-Flight Flow Visualization Obtained From the NASA High Alpha Research Vehicle," NASA TM-101734, January 1991.

${ }^{5}$ Murman, S.M., Rizk, Y.M, Cummings, R.M., and Schiff, L.B., "Computational Investigation of Slot Blowing for Fuselage Forebody Flow Control," Aircraft Design, Vol. 2, No. 1, 1999, pp. 45-63.

${ }^{6}$ Werlé, H., "Quelques résultats expérimentaux sur les ailes en flèche, aux faibles vitesses, obtenus en tunnel hydrodynamique," $L a$ Recherche Aéronautique, No. 41, Sep.-Oct. 1954, pp. 15-21.

${ }^{7}$ Peckham, D.H., Atkinson, S.A., "Preliminary Results of Low Speed Wind Tunnel Tests on a Gothic Wing of Aspect Ration 1.0," Aeronautical Research Council Technical Report, C.P. No 508, T.N. Aero 2504, Apr. 1957.

${ }^{8}$ Elle, B.J., "An Investigation at Low Speed of the Flow near the Apex of Thin Delta Wings with Sharp Leading Edges,” Aeronautical Research Council R\&M, No. 3176, 1961.

${ }^{9}$ Lambourne, N.C., Bryer, D.W., "The Bursting of Leading-Edge Vortices-Some Observations and Discussion of the Phenomenon," Aeronautical Research Council R\&M No. 3282, 1962.

${ }^{10}$ Hall, M.G., "Vortex Breakdown," Annual Review of Fluid Mechanics, Vol. 4, 1972, pp. 195-218.

${ }^{11}$ Leibovich, S., “The Structure of Vortex Breakdown,” Annual Review of Fluid Mechanics, Vol. 10, 1978, pp. 221-46.

${ }^{12}$ Sarpkaya, T. “On Stationary and Traveling Vortex Breakdowns,” Journal of Fluid Mechanics, Vol. 45, Part 3, 1971 , pp. $545-559$.

${ }^{13}$ Nelson, R.C., "Unsteady Aerodynamics of Slender Wings," Aircraft Dynamics at High Angles of Attack: Experiments and Modeling, AGARD- R-776, 1991, pp.1-1 to 1-26.

${ }^{14}$ Délery, J., “Aspects of Vortex Breakdown,” Progress in Aerospace Sciences, Vol. 30, No. 1, 1994, pp. 1 -59.

${ }^{15}$ Faller, J.H., Leibovich, S., “Disrupted States of Vortex Flow and Vortex Breakdown,” Physics of Fluids, Vol. 20, No. 9, 1977, pp.1385-1400.

${ }^{16}$ Menke, M., Yang, H., Gursul, I., "Experiments on the Unsteady Nature of Vortex Breakdown over a Delta Wing," Experiments in Fluids, Vol. 27, No. 3, 1999, pp. 262-272.

${ }^{17}$ Mitchell, A.M., Molton, P., Barberis, D., Délery, J., “Oscillation of Vortex Breakdown Location and Control of the Time-Averaged Location by Blowing," AIAA Journal, Vol. 38, No. 5, 2000, pp.793-803.

${ }^{18}$ Spalart, P. R., and Allmaras, S.R., “A One Equation Turbulence Model for Aerodynamic Flows,” La Recherche Aerospatiale, Vol. 1, 1994, pp. 5-21.

${ }^{19}$ Spalart, P., "Young-Person's Guide to Detached-Eddy Simulation Grids," NASA CR 2001-211032.

${ }^{20}$ Strang, W.Z., Tomaro, R.F., Grismer, M.J., "The Defining Methods of Cobalt: A Parallel, Implicit, Unstructured Euler/Navier- Stokes Flow Solver," AIAA Paper 99-0786, Jan. 1999. 
${ }^{21}$ Forsythe, J.R., Hoffmann, K.A., Cummings, R.M., Squires, K.D., "Detached-Eddy Simulation with Compressibility Corrections Applied to a Supersonic Axisymmetric Base,” Journal of Fluids Engineering, Vol. 124, No. 4, 2002, pp. 911-923.

${ }^{22}$ Morton, S.A., Forsythe, J.R., Mitchell, A.M., and Hajek, D., "Detached-Eddy Simulations and Reynolds-Averaged Navier-Stokes Simulations of Delta Wing Vortical Flowfields," Journal of Fluids Engineering, Vol. 124, No. 4, 2002, pp. 924-932.

${ }^{23}$ Squires, K.D., Forsythe, J.R., and Spalart, P.R., "Detached-Eddy Simulation of the Separated Flow Around a Forebody Cross-Section," Direct and Large Eddy Simulation IV, ERCOFTAC Series - Volume 8, B.J. Geurts, R. Friedrich and O. Metais, editors, Kluwar Academic Press, pp. 481-500, 2001.

${ }^{24}$ Forsythe, J.R., Squires, K.D., Wurtzler, K.E., and Spalart, P.R., "Detached-Eddy Simulation of Fighter Aircraft at High Alpha," Journal of Aircraft, Vol. 41, No. 2, 2004, pp. 193-200.

${ }^{25}$ Forsythe, J.R., Woodson, S.H., "Unsteady CFD Calculations of Abrupt Wing Stall Using Detached-Eddy Simulation,” AIAA Paper 2003-0594, Jan. 2003.

${ }^{26}$ Samareh, J., "Gridtool: A Surface Modeling and Grid Generation Tool,” Proceedings of the Workshop on Surface Modeling, Grid Generation, and Related Issues in CFD Solution, NASA CP-3291, 1995.

${ }^{27}$ Pirzadeh, S., "Progress Toward A User-Oriented Unstructured Viscous Grid Generator,” AIAA Paper 96-0031, Jan. 1996.

${ }^{28}$ Spalart, P. R. , Jou W-H. , Strelets M. , and Allmaras, S. R., "Comments on the Feasibility of LES for Wings, and on a Hybrid RANS/LES Approach,” Advances in DNS/LES, 1st AFOSR Int. Conf. on DNS/LES, Aug. 4-8, 1997, Greyden Press, Columbus Oh.

${ }^{29}$ Forsythe, J.R., Hoffmann, K.A., Dieteker, F.F., "Detached-Eddy Simulation of a Supersonic Axisymmetric Base Flow with an Unstructured Flow Solver," AIAA Paper 2000-2410, June 2000.

${ }^{30}$ Morton, S.A., Forsythe, J.R., Squires, K.D., and Wurtzler, K.E., “Assessment of Unstructured Grids for Detached-Eddy Simulation of High Reynolds Number Separated Flows,” 8 ${ }^{\text {th }}$ ISGG Conference, Honolulu, June 2002.

${ }^{31}$ Mitchell, A., Morton, S., and Forsythe, J., “Analysis of Delta Wing Vortical Substructures Using Detached-Eddy Simulation,” AIAA Paper 2002-2968, June 2002.

${ }^{32}$ Pirzadeh, S.Z., "Solution-Adaptive Unstructured Grid Method by Grid Subdivision and Local Remeshing," Journal of Aircraft, Vol. 37, No. 5, 2000, pp. 818-824.

${ }^{33}$ Squire, H.B., Jones, J.G., and Stanbrook, A., "An Experimental Investigation of the Characteristics of Some Plane and Cambered 65 Deg Delta Wings at Mach Numbers from 0.7 to 2.0,” ARC, R\&M No. 3305, 1961.

${ }^{34}$ Payne, F.M., Ng, T.T., Nelson, R.C. and Schiff, L.B., "Visualization and Wake Surveys of Vortical Flow over a Delta Wing," AIAA Journal, Vol. 26, No. 2, 1988, pp 137-143.

${ }^{35}$ Lowson, M.V., "Visualization Measurements of Vortex Flows," AIAA Paper 89-0191, Jan. 1989.

${ }^{36}$ Veerhagen, N.G., Meeder, J.P., and Verhelst, J.M., "Boundary Layer Effects of the Flow of a Leading-Edge Vortex," AIAA Paper 933463, Aug. 1993. 1994.

${ }^{37}$ Washburn, A.E. and Visser, K.D., "Evolution of Vortical Structures in the Shear Layer of Delta Wings,” AIAA Paper 94-2317, June

${ }^{38}$ Lowson, M.V., Riley, A.J., and Swales, C., "Flow Structure Over Delta Wings,” AIAA Paper 95-0586, Jan. 1989.

39 Honkan, A. and Andreopoulos, J., "Instantaneous Three-Dimensional Vorticity Measurements in Vortical Flow Over a Delta Wing," AIAA Journal, Vol. 35, No. 10, 1997, pp. 1612-1620.

${ }^{40} \mathrm{Ng}$, T.T. and Oliver, D.R., “Leading-Edge Vortex and Shear Layer Instabilities,” AIAA Paper 98-0313, Jan. 1998.

${ }^{41}$ Mitchell, A.M. and Molton, P., "Vortical Substructures in the Shear Layers Forming Leading-Edge Vortices," AIAA Journal, Vol. 40, No. 8, 2002, pp. 1689-1692.

42 Ames Research Center Dryden Flight Research Facility, "High Alpha Research Vehicle Phase II Flight Report, Flight 197- 199," HA94-70-660, Feb. 1994. 1189.

${ }^{43}$ Gee, K., Murman, S.M., and Schiff, L.B., “Computation of F/A-18 Tail Buffet,” Journal of Aircraft, Vol. 33, No. 6, 1996, pp. 1181- 
${ }^{44}$ Morton, S.A., Steenman, M.B., Cummings, R.M., and Forsythe, J.R., "DES Grid Resolution Issues for Vortical Flows on a Delta Wing and an F-18C," AIAA Paper 2003-1103, Jan. 2003.

${ }^{45}$ Sheta, E.F., “Alleviation of Vertical Tail Buffeting of F/A-18 Aircraft,” Journal of Aircraft, Vol. 41, No. 2, 2004 , pp. $322-330$.

${ }^{46}$ Gursul, I., "Review of Unsteady Vortex Flows Over Delta Wings,” AIAA Paper 2003-3942, June 2003.

${ }^{47}$ Ghaffari, F., "Navier-Stokes, Flight, and Wind Tunnel Flow Analysis for the F/A-18 Aircraft,” NASA TP 3478, Dec. 1994. 